Revista Brasileira de Cartografia

ISSN 1808-0936 | https://doi.org/10.14393/revbrascartogr

Sociedade Brasileira de Cartografia, Geodésia, Fotogrametria e Sensoriamento Remoto

\title{
Análise do GNSS PPP multi-constelações com uso dos sistemas GPS, GLONASS e Galileo
}

\author{
Analysis of GNSS PPP multi-constellations using GPS, GLONASS and Galileo systems
}

Raiane Rintielle Vaz Menezes ${ }^{1}$ e William Rodrigo Dal Poz ${ }^{2}$

1 Universidade Federal de Viçosa, Programa de Pós-Graduação em Eng. Civil, Área de concentração em Informações Espaciais,

Viçosa, Brasil. rintielle@gmail.com.

ORCID: https://orcid.org/0000-0001-6717-728X

2 Universidade Federal de Viçosa, Programa de Pós-Graduação em Eng. Civil, Área de concentração em Informações Espaciais, Viçosa, Brasil. william.dalpoz@ufv.br.

ORCID: https://orcid.org/0000-0001-9532-3643

Recebido: 06.2019| Aceito: 02.2020

Resumo: Dentre os sistemas que compõem o GNSS, estão completamente funcionais o GPS e o GLONASS. Além disso, encontram-se em desenvolvimento o Galileo e o BeiDou, sendo que o Galileo dispõe de 17 satélites operantes. Desta forma, o objetivo deste trabalho é investigar o desempenho de combinações multi-constelações envolvendo GPS, GLONASS e Galileo em quatro estações da RBMC, utilizando o método de posicionamento PPP. Para alcançar esse propósito, foram realizadas avaliações das acurácias planimétricas e altimétricas de estações multi-constelações, pertencentes à RBMC, abrangendo os 30 dias do mês de abril de 2018. Os dados foram editados no freeware GFZRNX a fim de obter arquivos com intervalos de 60 e 180 minutos de rastreio, contendo apenas dados GPS, GPS e GLONASS, GPS e Galileo e, a combinação dos três sistemas. Os dados foram processados a partir do software científico Bernese, versão 5.2. Os resultados mais acurados, tanto na planimetria quanto na altimetria, foram proporcionados pela tradicional integração GPS/GLONASS. Em segundo lugar, a combinação GPS/GLONASS/Galileo mostrou-se promissora, evidenciando sua interoperabilidade no método de posicionamento PPP.

Palavras-chave: GNSS. Multi-Constelações. PPP. Bernese.

\begin{abstract}
Among the systems that make up GNSS, GPS and GLONASS are fully functional. In addition, Galileo and BeiDou are under development, and Galileo has 17 operating satellites. Thus, the purpose of this paper is to investigate the performance of multi-constellation combinations such as GPS, GLONASS and Galileo in some RBMC stations, using the PPP positioning method. For this purpose, the planimetric and altimetric accuracy of four multi-constellation stations were evaluated, belonging to the RBMC, covering the 30 days of April 2018. The data were edited in the GFZRNX freeware in order to obtain files with 60 and 180 minutes tracking intervals, containing only GPS, GPS and GLONASS, GPS and Galileo and the combination of the three systems. Data were processed from Bernese scientific software, version 5.2. The most accurate results in planimetry and altimetry were provided by the traditional GPS/GLONASS integration. Second, the GPS/GLONASS/Galileo combination was promising, showing its interoperability in the PPP positioning method.
\end{abstract}

Keywords: GNSS. Multi-Constellation. PPP. Bernese.

\section{INTRODUÇÃO}

Tradicionalmente, o GPS (Global Positioning System) é a constelação mais conhecida e empregada do GNSS (Global Navigation Satellite Systems). Com a recuperação do GLONASS, em 2012, bem como as emergentes constelações Galileo e BeiDou, ambos previstos para estarem operacionais até o ano de 2020 (BEIDOU, 2018; ESA, 2018), implicam que o campo da navegação por satélite está passando por mudanças consideráveis. A integração entre estes sistemas aumentará significativamente o número de satélites observados, otimizando a geometria espacial, melhorando, consequentemente, a confiabilidade do 
posicionamento (LI et al., 2015).

Quando os quatro sistemas estiverem totalmente implementados, cerca de 120 satélites de navegação estarão operacionais, contribuindo para uma melhor geometria entre os satélites e resultando em estimativas superiores de posicionamento. Sem dúvida, o mundo da navegação por satélite está passando por muitas mudanças com o rápido desenvolvimento do GNSS multi-constelações, potencializando aplicações e as tornando cada vez mais precisas e confiáveis (LI et al., 2015).

Diante dessas mudanças, o IGS (International GNSS Service) ampliou suas pesquisas, e em meados de 2011 iniciou o projeto MGEX (Multi-GNSS Experiment). O qual foi criado para coletar e analisar dados para determinar as órbitas precisas combinadas e outras correções, com o intuito de explorar e promover o uso das novas observações multi-constelações em conjunto (MONTENBRUCK et al., 2017).

A RBMC (Rede Brasileira de Monitoramento Contínuo dos Sistemas GNSS) é a rede principal de referência ativa no Brasil, a qual disponibiliza gratuitamente dados GNSS, informações das estações, bem como coordenadas de referência no sítio do IBGE. Esta rede encontra-se em fase de ampliação e modernização, no qual visa expandir o fornecimento de dados GNSS, principalmente dados das constelações emergentes, Galileo e BeiDou. Atualmente a RBMC conta com 20 estações equipadas com receptores multi-constelações, fornecendo dados RINEX 3.0 a partir do mês de agosto de 2018 (COSTA et al., 2018).

Diante disso, a literatura científica internacional apresenta alguns estudos sobre a interoperabilidade dos sistemas GNSS com dados simulados, como os trabalhos de Ji et al. (2010), Odijk e Teunissen (2013) e, dados reais de Li et al. (2015), Pan et al. (2016) e Tarantino et al. (2018). Porém, no Brasil têm-se poucos estudos relacionados, como o de Setti Júnior, Alves e Gouveia (2016) que compararam a contribuição do Galileo integrado ao GPS no posicionamento por ponto simples. Verificaram uma melhora no posicionamento ao considerar a combinação entre as constelações. No entanto, em várias ocasiões a integração entre o GPS e Galileo não proporcionou bons resultados. Já Setti Júnior e Alves (2017) avaliaram a geometria dos satélites e a acurácia do posicionamento com o uso de dados combinados GPS e Galileo, em comparação com o uso de dados GPS, além da qualidade da geometria dos satélites visíveis (PDOP). Constataram que houve uma melhora tanto na estimativa do posicionamento, ao utilizar dados das duas constelações, quanto na geometria dos satélites. Ambos os estudos utilizaram apenas dados de uma frequência, ou seja, portadora L1 (GPS) e E1 (Galileo).

Conforme discutido, pode-se observar uma carência de estudos sobre a atual contribuição da constelação Galileo para o posicionamento multi-constelações no Brasil. Fato que justificaria pesquisas desta natureza. Além disso, a situação atual do GNSS é muito diferente em relação à época dos estudos descritos. É importante destacar que há uma ausência de softwares comerciais, que já incluam em seus processamentos os novos sistemas que compõem o GNSS como o Galileo (SETTI JÚNIOR, ALVES e GOUVEIA, 2016). O software científico BERNESE GNSS (BSW), que é conhecido pela comunidade acadêmica, por fornecer um alto padrão de qualidade para aplicações geodésicas, dispõe de um pacote capaz de processar dados Galileo integrado com GPS e/ou GLONASS, viabilizando estudos com esses sistemas. Deve-se destacar que este pacote se encontra em estágio experimental e disponível para utilização na versão 5.2 do BSW (DACH e FRIDEZ, 2017).

Apesar de não estar totalmente operacional, nos dias de hoje o sistema Galileo dispõe de 17 satélites operantes (GSC, 2018). Desta forma, é um momento oportuno para pesquisas relacionadas a integração dos sistemas GNSS. Visto que, no Brasil, nenhum estudo avaliou a acurácia na integração entre Galileo, GLONASS e GPS em mais de uma estação multi-constelação.

Desta forma, o presente trabalho tem por objetivo investigar o desempenho de combinações multiconstelações envolvendo GPS, GLONASS e Galileo. Para esse fim, realizou-se uma análise da acurácia (tomando como referência as coordenadas oficiais dos respectivos descritivos), de quatro estações multiconstelações da RBMC, considerando intervalos de rastreio curto e longo (60 e 180 minutos) com a técnica PPP (Posicionamento por Ponto Preciso) no BSW.

\section{SIMILARIDADE E DIFERENÇAS ENTRE GPS, GLONASS E GALILEO}

O sistema Galileo teve sua origem para civis, diferente do GPS e GLONASS que inicialmente foram 
propostos para fins militares. Apesar desses sistemas fazerem parte do GNSS, cada constelação também apresenta diferenças e similaridades técnicas. A Tabela 1 sumariza uma comparação entre os sistemas GPS, GLONASS e Galileo.

Tabela 1 - Resumo das características do GPS, GLONASS e Galileo $\left({ }^{1} 24\right.$ principais e 7 extras; ${ }^{2} 17$

\begin{tabular}{|c|c|c|c|c|}
\hline \multicolumn{5}{|c|}{ operacionais). } \\
\hline Caracteristicas & GPS & \multicolumn{2}{|c|}{ GLONASS } & Galileo \\
\hline Número de satélites & $24^{1}$ & \multicolumn{2}{|c|}{24} & $30^{2}$ \\
\hline Planos orbitais & 6 & \multicolumn{2}{|c|}{3} & 3 \\
\hline Inclinação da órbita & $55^{\circ}$ & \multicolumn{2}{|c|}{$64,8^{\circ}$} & $56^{\circ}$ \\
\hline Altitude & $20.200 \mathrm{~km}$ & \multicolumn{2}{|c|}{$19.100 \mathrm{~km}$} & $23.222 \mathrm{~km}$ \\
\hline Período orbital & $11 \mathrm{~h} 58 \mathrm{~min}$ & \multicolumn{2}{|c|}{$11 \mathrm{~h} 15 \mathrm{~min}$} & $\sim 14 \mathrm{~h}$ \\
\hline Sistema de Referência & WGS-84 & \multicolumn{2}{|c|}{ PZ-90.11 } & GTRF \\
\hline Sistema de Tempo & UTC (USNO) & \multicolumn{2}{|c|}{ UTC (SU) } & GST \\
\hline Tipo de Sinal & CDMA & FDMA & CDMA & CDMA \\
\hline \multirow{3}{*}{ Frequências (MHz) } & L1: $1.575,42$ & $\begin{array}{c}\text { G1: } 1.602,00+ \\
k^{*} 0,5625\end{array}$ & E1: $1.575,42$ & E1: $1.575,42$ \\
\hline & L2: $1.227,60$ & $\begin{array}{c}\text { G2: } 1.246,00+ \\
\mathrm{k}^{*} 0,4375\end{array}$ & E6: $1.278,75$ & E6: $1.278,75$ \\
\hline & L5: $1.176,45$ & & E5: $1.191,79$ & E5: $1.191,79$ \\
\hline
\end{tabular}

Fonte: Os autores (2020).

Em relação à estrutura dos sinais, os satélites dos sistemas Galileo e GPS, utilizam a técnica CDMA (Code Division Multiple Access), em que todos os satélites transmitem as mesmas frequências, e a identificação destes é dada pelo PRN (Pseudo Random Noise) atribuído a cada satélite (MONICO, 2008). A concepção original de transmissão de sinais no GLONASS dá-se pela técnica denominada FDMA (Frequency Division Multiple Access), onde cada satélite transmite na sua própria frequência e, dessa forma, podendo identificálos. No entanto, com a modernização dos novos satélites GLONASS, em 2011, o sistema passou a utilizar, também, a técnica CDMA. Deste modo, facilitando a compatibilidade e a interoperabilidade com os demais sistemas.

O sistema de tempo GPS está relacionado ao TAI (International Atomic Time) e é referenciado ao UTC (Universal Coordinated Time) mantido pelo United States Naval Observatory (UTC [USNO]). O tempo GPS não é incrementado pelos saltos de segundos inteiros (leap seconds) e a diferença com o TAI é de 19 segundos (SEEBER, 2003). O GLONASS tem seu sistema de tempo baseado em relógios de hidrogênio, considera os saltos de segundos e possui um deslocamento constante de três horas, devido à diferença de fusos entre Moscou (+3h) e Greenwich (HOFMANN-WELLENHOF, LICHTENEGGER e COLLINS, 2008). O Sistema de Tempo Galileo (GST) é mantido pelos GCCs (Galileo Control Centers), localizados na Itália e Alemanha, onde são geradas as correções em relação ao UTC com precisão de 28 bilionésimos de segundos (ESA, 2018).

Para definir as órbitas de seus satélites, cada constelação GNSS apresenta seu próprio sistema de referência. Para o GPS, o sistema de referência utilizado é o WGS-84 (World Geodetic System) que se encontra na realização (G1762) (FIG, 2018). O GLONASS faz uso, atualmente, do PZ-90.11 (Parametry Zemli 1990) (UNOOSA, 2018a), enquanto o Galileo utiliza hoje em dia o GTRF (Galileo Terrestrial Reference Frame) na sua realização GTRF 18v01 (UNOOSA, 2018b). Os três sistemas são geocêntricos e muito similares, além de serem consistentes com o ITRF (International Terrestrial Reference Frame), porém existem pequenas diferenças, centimétricas, em suas definições (HOFMANN-WELLENHOF, LICHTENEGGER e COLLINS, 2008). Essas diferenças devem ser consideradas na integração entre os sistemas, pois influenciam nas coordenadas do satélite. Por conseguinte, as coordenadas dos satélites devem ser transformadas para um sistema em comum, antes de se aplicar o ajustamento. Os parâmetros de transformação entre os diferentes sistemas de coordenadas são dados de acordo com a transformação de Helmert. Maiores detalhes podem ser encontrados, por exemplo, em Hofmann-Wellenhof, Lichtenegger e Collins (2008).

Para propósitos de posicionamento multi-constelações com a metodologia PPP, é indispensável que as coordenadas das diferentes constelações GNSS estejam referenciadas à mesma realização. Para isso, utiliza-se as efemérides precisas disponibilizadas pelo Projeto MGEX, que são referenciadas, atualmente, ao IGS14. Assim, as coordenadas estimadas pelo PPP também serão referenciadas ao IGS14 (IGS, 2018). 
Da mesma forma que as efemérides precisas MGEX são fornecidas no mesmo referencial (ITRF14/IGS14), os erros dos relógios dos satélites GNSS também devem ter uma escala de tempo (time scale) em comum. Os produtos MGEX consideram como referência o sistema de tempo GPS (AFIFI e ELRABBANY, 2015). Desta forma, os erros dos relógios MGEX dos satélites GLONASS e Galileo são referenciados ao sistema de tempo GPS (caso similar ocorre com o uso do BeiDou). Verifica-se, portanto, a relevante importância do projeto MGEX na exequibilidade do PPP multi-constelações.

Evidencia-se que a interoperabilidade é um conceito importante no GNSS, podendo ser definida como a capacidade de usar dois ou mais serviços em conjunto para conseguir melhor performance ao nível do usuário (VENTORIM e DAL POZ, 2016). Desta forma, apesar das diferenças técnicas entre os sistemas, a similaridade das frequências centrais que eles usam, cria o potencial para garantir a interoperabilidade de um receptor GNSS.

\section{MATERIAIS E MÉTODOS}

\subsection{Materiais}

Nesta pesquisa foram utilizados dados GNSS de 4 estações da RMBC (3 em São Paulo e 1 em Minas Gerais), levando em conta o maior número de satélites Galileo rastreados na época escolhida. A fim de possibilitar uma análise temporal, foram obtidos dados de 30 dias, referentes ao mês de abril de 2018. Além disso, pretendeu-se avaliar a relação da acurácia com o tempo de rastreio empregando dois intervalos, um curto (60 min) e um longo (180 min). Vale salientar que o menor intervalo foi utilizado em função do BSW não processar dados Galileo com intervalo inferior a 60 minutos, já o intervalo de 180 minutos foi considerado por se tratar de um tempo aproximado para utilização dos dados como estação base, para fins de processamento relativo.

Em sequência, têm-se ilustrado a localização (Figura 1) e as informações dos receptores e antenas (Quadro 1) das estações selecionadas.

Figura 1 - Localização das estações selecionadas.

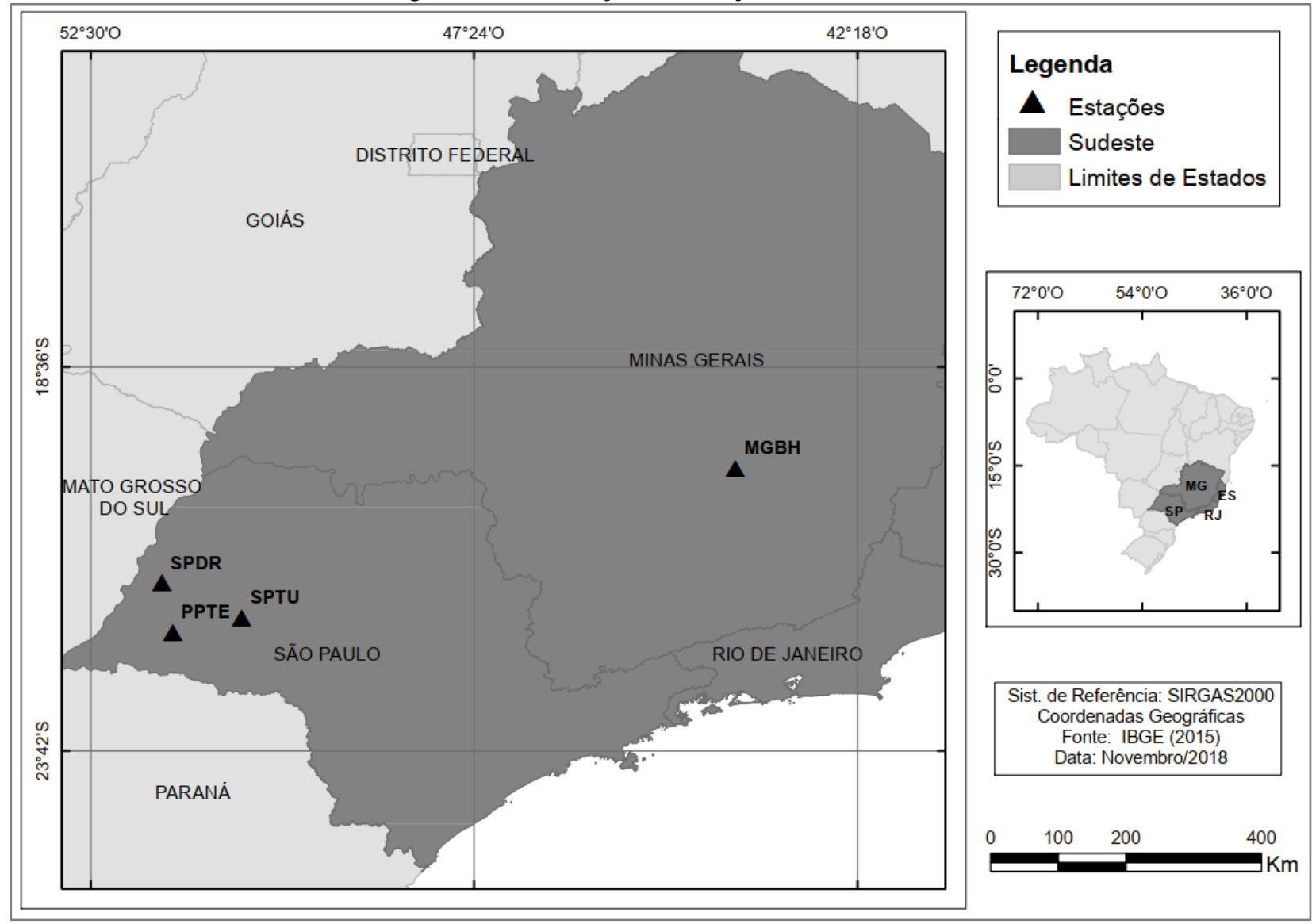

Fonte: Os autores (2020). 
Quadro 1 - Informações sobre os receptores e antenas das estações.

\begin{tabular}{|c|c|c|c|c|}
\hline Estação & Tipo do Receptor & Data de Instalação & Tipo de Antena & Data de Instalação \\
\hline PPTE & TRIMBLE NETR9 & $18 / 03 / 2014$ & GNSS CHOKE RING (TRM59800.00) & $07 / 09 / 2009$ \\
\hline SPTU & TRIMBLE NETR9 & $25 / 07 / 2014$ & $\begin{array}{c}\text { ZEPHYR GNSS GEODETIC II } \\
\text { (TRM57971.00) }\end{array}$ & $25 / 07 / 2014$ \\
\hline SPDR & TRIMBLE NETR9 & $25 / 07 / 2014$ & $\begin{array}{c}\text { ZEPHYR GNSS GEODETIC II } \\
\text { (TRM57971.00) }\end{array}$ & $25 / 07 / 2014$ \\
\hline MGBH & TRIMBLE NETR9 & $20 / 03 / 2018$ & $\begin{array}{c}\text { ZEPHYR 3 GEODETIC } \\
\text { (TRM115000.00) }\end{array}$ & $17 / 09 / 2008$ \\
\hline
\end{tabular}

Fonte: Os autores (2020).

\subsection{Métodos}

O fluxograma representado na Figura 2 apresenta, em resumo, os principais passos da metodologia empregada nesta pesquisa.

Figura 2 - Fluxograma da metodologia aplicada no presente estudo.

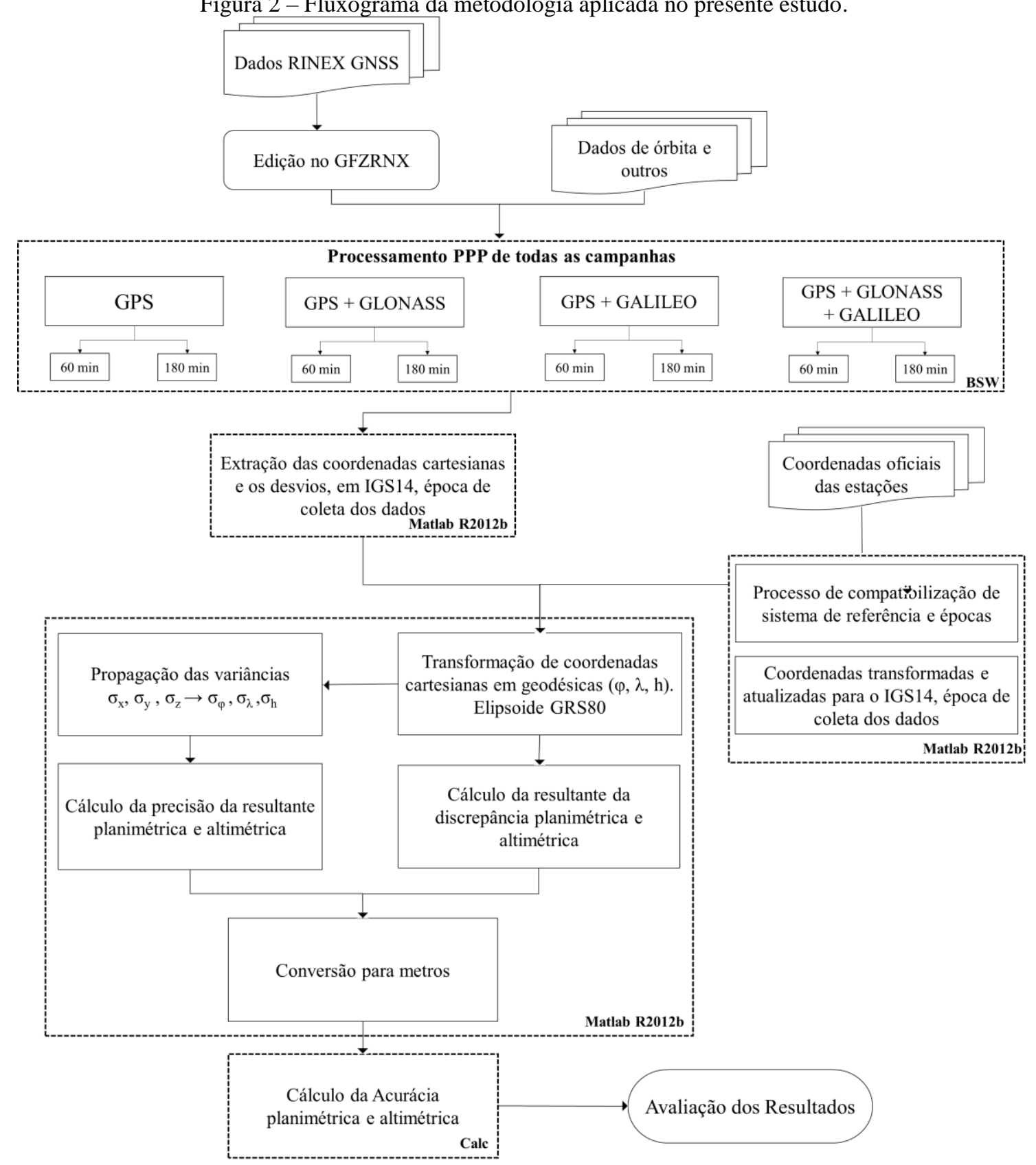

Fonte: Os autores (2020). 
As observáveis GNSS foram obtidas no sítio da RBMC, onde são disponibilizados arquivos no formato RINEX (Receiver Independent Exchange Format) com 24h de observações, para cada dia e para cada estação selecionada. Foi necessário realizar a transformação dos arquivos RINEX da versão 2.11 para a versão 3.01, fazendo-se uso do freeware GFZRNX (RINEX GNSS Data Conversion and Manipulation Toolbox), desenvolvido pela GFZ Data Services (NISCHAN, 2016). De acordo com Dach e Fridez (2017), as observáveis do sistema Galileo são reconhecidas pelo BSW somente na versão 3 do RINEX, justificando-se, assim, a transformação de versões dos arquivos. Somente a partir de agosto de 2018 os dados da RBMC passaram a ser fornecidos no formato RINEX versão 3.0 (COSTA et al., 2018).

À vista disso, os arquivos foram editados no freeware GFZRNX, admitindo apenas dados GPS, GPS e GLONASS, GPS e Galileo e os dados dos três sistemas em conjunto: GPS, GLONASS e Galileo. Em todos os casos foram empregados dois intervalos de rastreio (60 e $180 \mathrm{~min}$ ), todos a partir das $12 \mathrm{~h}$ UTC.

O processamento das observáveis GNSS foi realizado no software científico Bernese, versão 5.2, desenvolvido pelo Instituto Astronômico da Universidade de Berna (AIUB) - Suíça. Para realizar o processamento dos dados neste software, vários arquivos devem ser obtidos, como consta na Quadro 2.

Quadro 2 - Produtos de correções e seu local de obtenção.

\begin{tabular}{|c|c|}
\hline Arquivos de correções & Local de Obtenção \\
\hline $\begin{array}{c}\text { Soluções finais de órbitas, correções de relógio dos satélites e } \\
\text { parâmetros de orientação da Terra }\end{array}$ & $\begin{array}{c}\text { Multi-constelação Extension (MGEX) do IGS } \\
\text { (ftp://ftp.aiub.unibe.ch/CODE_MGEX/CODE) }\end{array}$ \\
\hline $\begin{array}{c}\text { DCBs (Differential Code Biases) e } \\
\text { Mapas ionosféricos }\end{array}$ & Center for Orbit Determination in Europe (CODE) \\
\hline Parâmetros do modelo de cargas oceânicas & (http://holt.oso.chalmers.se/loading) \\
\hline $\begin{array}{c}\text { Carga de pressão atmosférica (modelo de Ray e Ponte (2003), } \\
\text { Modelo troposférico (GPT/GMF) e Correções de Marés } \\
\text { Terrestres (IERS - 2010) }\end{array}$ & Foram gerados empregando o próprio BSW \\
\hline
\end{tabular}

Fonte: Os autores (2020).

Em seguida, a partir de trinta e duas campanhas no BSW (8 campanhas x 4 estações) foi realizado o processamento dos dados empregando a técnica PPP. Ressaltando que para todos os processamentos foi utilizado intervalo das observações de 15 segundos e máscara de elevação de $10^{\circ}$. Foi aplicada a combinação linear livre dos efeitos da ionosfera, eliminando, desta forma, os efeitos de primeira ordem da ionosfera. A troposfera foi modelada a partir do modelo GPT (Global Pressure Temperature)/GMF (Global Mapping Function), de acordo com o recomendado pelo manual do BSW.

Como resultado, o BSW gera diversos arquivos contendo várias informações sobre o processamento, assim como as coordenadas cartesianas $(\mathrm{X}, \mathrm{Y}, \mathrm{Z})$ e seus respectivos desvios-padrão $\left(\sigma_{\mathrm{x}}, \sigma_{\mathrm{y}}, \sigma_{\mathrm{z}}\right)$ no sistema de referência ITRF2014 (IGS14), na época de coleta dos dados. Devido ao grande volume de dados gerados pelos processamentos no BSW, empregou-se uma rotina em Matlab, a fim de automatizar o processo de extração das coordenadas e seus respectivos desvios.

As coordenadas de referência contidas no descritivo das estações da RBMC se encontram no sistema de referência oficial do Brasil, denominado SIRGAS2000, época 2000,4, o qual é uma densificação regional do ITRF2000 na época 2000,4 (SIRGAS, 2018). Por isso, é necessária a realização da transformação de referencial do SIRGAS2000 (ITRF2000) para ITRF2014 (IGS14), tal como a atualização de coordenadas para compatibilizar a época de referência $(2000,4)$ para a época do levantamento dos dados $(2018,28$ - referente ao dia 15 do mês de abril). Desta forma, as coordenadas a serem comparadas estarão referenciadas na mesma época e mesma realização do sistema de referência.

$\mathrm{Na}$ atualização de coordenadas é necessário considerar as componentes de velocidade para cada estação envolvida. Portanto, as componentes do vetor velocidade das estações $\left(\mathrm{V}_{\mathrm{x}}, \mathrm{V}_{\mathrm{y}}, \mathrm{V}_{\mathrm{z}}\right)$ foram obtidas através da aplicação VMS2009, a qual utiliza o VEMOS2009 (Velocity Model for SIRGAS) que é o modelo oficial do Sistema de Referencia Geodésico das Américas. Este modelo fornece as velocidades referenciadas ao ITRF2005 (DREWES e HEIDBACH, 2012), sendo assim, necessário transformar o referencial do modelo das velocidades do ITRF2005 para o ITRF2008 e, posteriormente, do ITRF2008 para o ITRF2014. A Figura 3 ilustra o esquema de transformação de referencial e atualização das coordenadas de referência. Detalhes sobre este procedimento podem ser encontrados em Braga e Dal Poz (2017). 
Figura 3 - Esquema de atualização e transformação das coordenadas de referência.

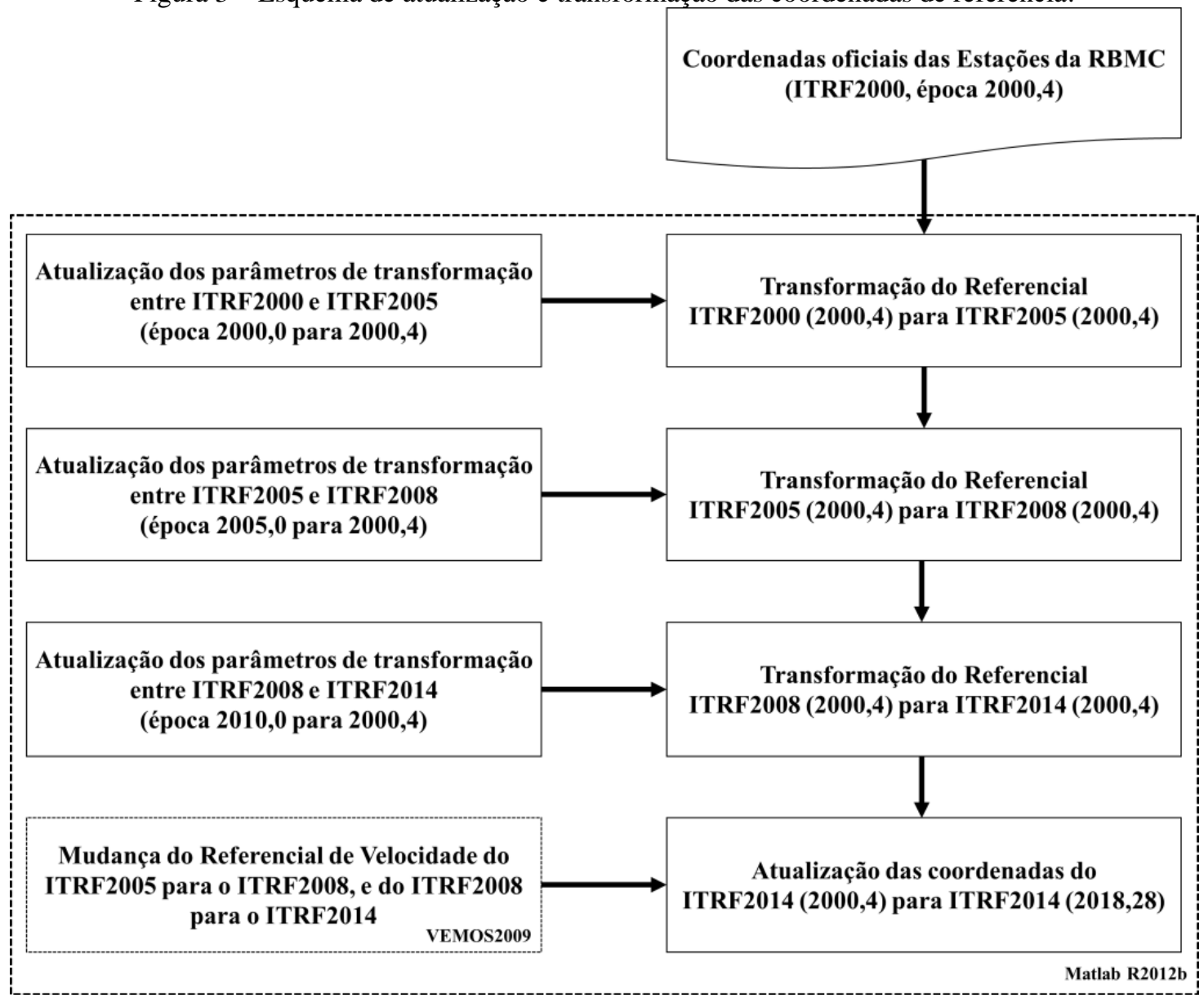

Fonte: Braga e Dal Poz (2017).

O modelo matemático utilizado para atualizar e transformar as coordenadas foi o recomendado pelo IERS, e disponibilizado pelo ITRF (2018). Já os parâmetros de transformação empregados foram os recomendados por Altamimi et al. (2007), Altamimi, Collilieux e Métivier (2012) e Altamimi et al. (2016). Esses parâmetros permitem transformar o referencial e atualizar as coordenadas entre as várias materializações do ITRF. Ressaltando que nesta pesquisa foi considerada a compatibilidade entre SIRGAS2000 e ITRF2000 (WESTON e SOLER, 2012), e entre o ITRF2014 e o IGS14 (REBISCHUNG e SCHMID, 2016).

Após a compatibilização entre referenciais e épocas, foram calculadas as acurácias planimétricas e altimétricas. A análise dos resultados foi realizada em coordenadas geodésicas. Salienta-se, que tanto as coordenadas estimadas quanto as de referência, já convertidas e transformadas para a época de coleta dos dados, são coordenadas cartesianas (X, Y, Z). Desta forma, essas coordenadas foram convertidas para coordenadas geodésicas $(\varphi, \lambda, h)$, através do método direto, proposto por Bowring (1976). Para a transformação foram utilizados os parâmetros do elipsoide GRS80 (PETIT e LUZUM, 2010), conforme recomenda a nota técnica $n^{\circ} 36$ do IERS Conventions 2010. Os cálculos, incluindo a transformação, foram implementados no Matlab.

De posse das coordenadas estimadas (BSW) e de referência $(\varphi, \lambda, h)$, realizou-se o cálculo das discrepâncias que foram obtidas a partir das coordenadas estimadas no BSW e as coordenadas tomadas como referência, disponibilizadas pelo IBGE em seus respectivos descritivos, transformadas e atualizadas. Para o cálculo das discrepâncias da resultante planimétrica $\left(\mathrm{D}_{\mathrm{P}}\right)$, foram utilizadas as seguintes equações:

$$
\left|\begin{array}{c}
\Delta_{\varphi(\text { radianos })} \\
\Delta_{\lambda(\text { radianos })}
\end{array}\right|=\left|\begin{array}{lll}
\varphi_{(\text {estimada radianos })} & - & \varphi_{(\text {referência radianos })} \\
\lambda_{(\text {estimada radianos })} & - & \lambda_{(\text {referência radianos })}
\end{array}\right|
$$

na Eq. (1), obtém-se as discrepâncias em radianos, fazendo-se necessária a conversão de radianos para metros, conforme a Eq. (2) (TORGE, 2001): 


$$
\left|\begin{array}{c}
\Delta_{\varphi(\text { metros })} \\
\Delta_{\lambda(\text { metros })}
\end{array}\right|=\left|\begin{array}{c}
\mathrm{M} \times \Delta_{\varphi(\text { radianos })} \\
\mathrm{N} \times \cos \varphi \times \Delta_{\lambda(\text { (radianos })}
\end{array}\right|
$$

sendo $\mathrm{M}$ o raio de curvatura da seção meridiana e $\mathrm{N}$ o raio de curvatura da seção primeiro vertical, conforme o conjunto de Eq. (3).

$$
\mathrm{M}=\frac{\mathrm{a}\left(1-\mathrm{e}^{2}\right)}{\left(1-\mathrm{e}^{2} \operatorname{sen}^{2} \varphi\right)^{\frac{3}{2}}} \quad \mathrm{~N}=\frac{\mathrm{a}}{\left(1-\mathrm{e}^{2} \operatorname{sen}^{2} \varphi\right)^{\frac{1}{2}}} \quad \mathrm{e}^{2}=\frac{\mathrm{a}^{2}-\mathrm{b}^{2}}{\mathrm{a}^{2}}
$$

onde: $\mathrm{e}^{2}$ é o quadrado da primeira excentricidade; $\varphi$ é a latitude média calculada em função das coordenadas estimadas e de referência; a é o semieixo maior do GRS80 e b o semieixo menor do GRS80. Logo, a discrepância planimétrica $\left(\mathrm{D}_{\mathrm{P}}\right)$ em metros é dada pela Eq. (4):

$$
\mathrm{D}_{\mathrm{P}}=\sqrt{\Delta_{\varphi(\text { metros })}{ }^{2}+\Delta_{\lambda(\text { metros })}{ }^{2}}
$$

a discrepância altimétrica $\left(\Delta_{\mathrm{h}}\right)$, em metros, é calculada conforme a Eq. (5):

$$
\Delta_{\mathrm{h}(\text { metros })}=\mathrm{h}_{(\text {estimado })}-\mathrm{h}_{(\text {referência })}
$$

para o cálculo das precisões é indispensável ter os desvios-padrão em coordenadas geodésicas, porém, o BSW fornece no arquivo SINEX (Solution INdependent EXchange format) apenas os desvios-padrão para as coordenadas cartesianas (X, Y, Z). Desta forma, foi necessário propagar a variância e a covariância das coordenadas geodésicas cartesianas para que fossem obtidos os desvios-padrão propagados para coordenadas geodésicas elipsoidais $(\varphi, \lambda, h)$. Na sequência, as precisões planimétricas ( $\sigma p)$ foram calculadas conforme Eq. (6):

$$
\sigma_{\mathrm{P}}=\sqrt{\sigma_{\varphi(\text { metros })^{2}}+\sigma_{\lambda(\text { metros })}^{2}}
$$

ressaltando que a precisão altimétrica é o próprio $\sigma_{\mathrm{h}}$. Desta forma, foram calculadas as acurácias planimétricas (Ap) e altimétricas $\left(\mathrm{A}_{\mathrm{h}}\right)$ utilizando as Eq. (7) e (8) (MIKHAIL e ACKERMAN, 1976; MONICO et al., 2009).

$$
\begin{aligned}
& A_{P}=\sqrt{D_{P(\text { metros })^{2}+\sigma_{P(\text { metros })}^{2}}} \\
& A_{h}=\sqrt{\Delta_{h \text { (metros })^{2}+\sigma_{h(\text { metros })}{ }^{2}}}
\end{aligned}
$$

de posse das acurácias, foram elaborados histogramas, com nove classes de intervalos regulares, da seguinte forma: $0,0-1,0 \mathrm{~cm}, 1,0-2,0 \mathrm{~cm}, \ldots, 8,0-9,0 \mathrm{~cm}$. As classes foram escolhidas de acordo com o resultado menos acurado $(8,3 \mathrm{~cm})$. Os histogramas apresentam os resultados em ordem crescente da esquerda para a direita. Assim, os resultados mais acurados se referem aos valores mais concentrados à esquerda e os menos acurados mais concentrados à direita dos histogramas. Contudo, visualmente, pode ser difícil analisar qual solução é mais acurada. Desta forma, foi proposto um índice para análise das acurácias, com base nos resultados apresentados nos histogramas. Para isso, realizou-se uma ponderação considerando o número de classes dos histogramas, que foi padronizado em 9 . Assim, a primeira classe $(0,0-1,0 \mathrm{~cm})$ recebe um valor maior de ponderação, que vai diminuindo, proporcionalmente, até o valor da última classe, no caso a nona classe (8,0$9,0 \mathrm{~cm}$ ). Os valores de ponderação, bem como os intervalos de acurácias são apresentados na Tabela 2. 
Tabela 2 - Fatores de ponderação para cada classe.

\begin{tabular}{c|c}
\hline Intervalos de Acurácias (cm) & Fator de Ponderação \\
\hline $0,0<\mathrm{x} \leq 1,0$ & 1 \\
\hline $1,0<\mathrm{x} \leq 2,0$ & $1 / 2$ \\
\hline $2,0<\mathrm{x} \leq 3,0$ & $1 / 3$ \\
\hline $3,0<\mathrm{x} \leq 4,0$ & $1 / 4$ \\
\hline $4,0<\mathrm{x} \leq 5,0$ & $1 / 5$ \\
\hline $5,0<\mathrm{x} \leq 6,0$ & $1 / 6$ \\
\hline $6,0<\mathrm{x} \leq 7,0$ & $1 / 7$ \\
\hline $7,0<\mathrm{x} \leq 8,0$ & $1 / 8$ \\
\hline $8,0<\mathrm{x} \leq 9,0$ & $1 / 9$ \\
\hline
\end{tabular}

Fonte: Os autores (2020).

De acordo com a Tabela 2, o limite superior da primeira classe é igual a 1,0 cm, enquanto o limite superior da última classe é igual a $9,0 \mathrm{~cm}$, ou seja, pode-se dizer que a última classe é nove vezes menos acurada em relação à primeira classe. Desta forma, a primeira classe (a mais acurada) recebe o maior valor de ponderação (1), que vai diminuindo, proporcionalmente, até a nona classe (1/9). Assim, foi adotada a média ponderada para analisar as acurácias estimadas. Esta média, foi nomeado como Fator de Acurácia (FA) e é apresentada pela Eq. (9), em que $C_{n}$ corresponde ao valor de ocorrência de acurácia na classe $n(1,2, \ldots, 9)$.

$$
\mathrm{FA}=\frac{\left(\left(C_{1} * 1\right)+\left(C_{2} * \frac{1}{2}\right)+\left(C_{3} * \frac{1}{3}\right)+\left(C_{4} * \frac{1}{4}\right)+\left(C_{5} * \frac{1}{5}\right)+\left(C_{6} * \frac{1}{6}\right)+\left(C_{7} * \frac{1}{7}\right)+\left(C_{8} * \frac{1}{8}\right)+\left(C_{9} * \frac{1}{9}\right)\right)}{\left(1+\frac{1}{2}+\frac{1}{3}+\frac{1}{4}+\frac{1}{5}+\frac{1}{6}+\frac{1}{7}+\frac{1}{8}+\frac{1}{9}\right)}
$$

diante disso, verifica-se que a acurácia de cada solução será diretamente proporcional ao FA. Portanto, quanto maior o valor do FA, maior será a acurácia. Assim, o FA terá 10,60 como o valor máximo (considerando o resultado ideal em que todos os valores de acurácias (30) estejam concentrados na primeira classe) e o pior valor como 1,18 (com todos valores de acurácia concentrados na última classe). Devido a estação SPTU contar com apenas 28 dias de observações, foi empregado um fator de padronização da escala. Para isto, bastou usar os FAs calculados para estação SPTU e multiplicar pela fração 30/28. Ressalta-se que todas as outras estações contavam com 30 dias de observações.

\section{RESULTADOS E ANÁLISES}

Com a finalidade de facilitar a identificação dos resultados apresentados nas figuras e tabelas, adotouse uma padronização da nomenclatura dos processamentos. Serão consideradas as iniciais referentes a cada sistema original, conforme a convenção adotada nos arquivos RINEX e MGEX (GPS-G; GLONASS-R e Galileo-E). Desta forma, para os processamentos dos sistemas GPS/GLONASS serão atribuídas as letras GR; para GPS/Galileo será GE; e por fim, para GPS/Galileo/GLONASS será GER. Todos os gráficos foram construídos com escala em unidade centimétrica. Nos histogramas unificou-se os dados referentes as classes de 7, 8 e 9 cm em uma única classe (>7), para melhor visualização dos resultados.

Os resultados referentes às resultantes planimétricas das quatro estações são ilustrados através das Figuras 4, 5 e da Tabela 3.

A partir dos gráficos de dispersão das acurácias planimétricas, ilustrados na Figura 4, pode-se verificar a alteração entre os valores das acurácias nos diferentes intervalos de rastreio, ou seja, quanto maior o intervalo melhor é a acurácia resultante. Situação atípica identificou-se em MGBH, nas combinações GR e GER, em que o aumento no tempo de rastreio proporcionou uma melhora na acurácia dos resultados.

Em conformidade com as figuras apresentadas e considerando todas as combinações e intervalos, a ocorrência de valores de acurácias melhores ou iguais a $1,0 \mathrm{~cm}$ foram identificadas em $93,33 \%$ na estação PPTE e somente $0,41 \%$ na estação SPDR. Isso permite inferir que PPTE se destacou e SPDR apresentou os resultados menos acurados entre as demais estações. Além disso, somente a estação PPTE proporcionou as maiores ocorrências de valores de acurácia pertencentes a primeira classe (acurácia milimétrica) em todas as outras análises, sendo 27 na combinação GR e 26 na GER, ambos no intervalo de 180 minutos. 
Figura 4 - Gráficos de dispersão da acurácia planimétrica.
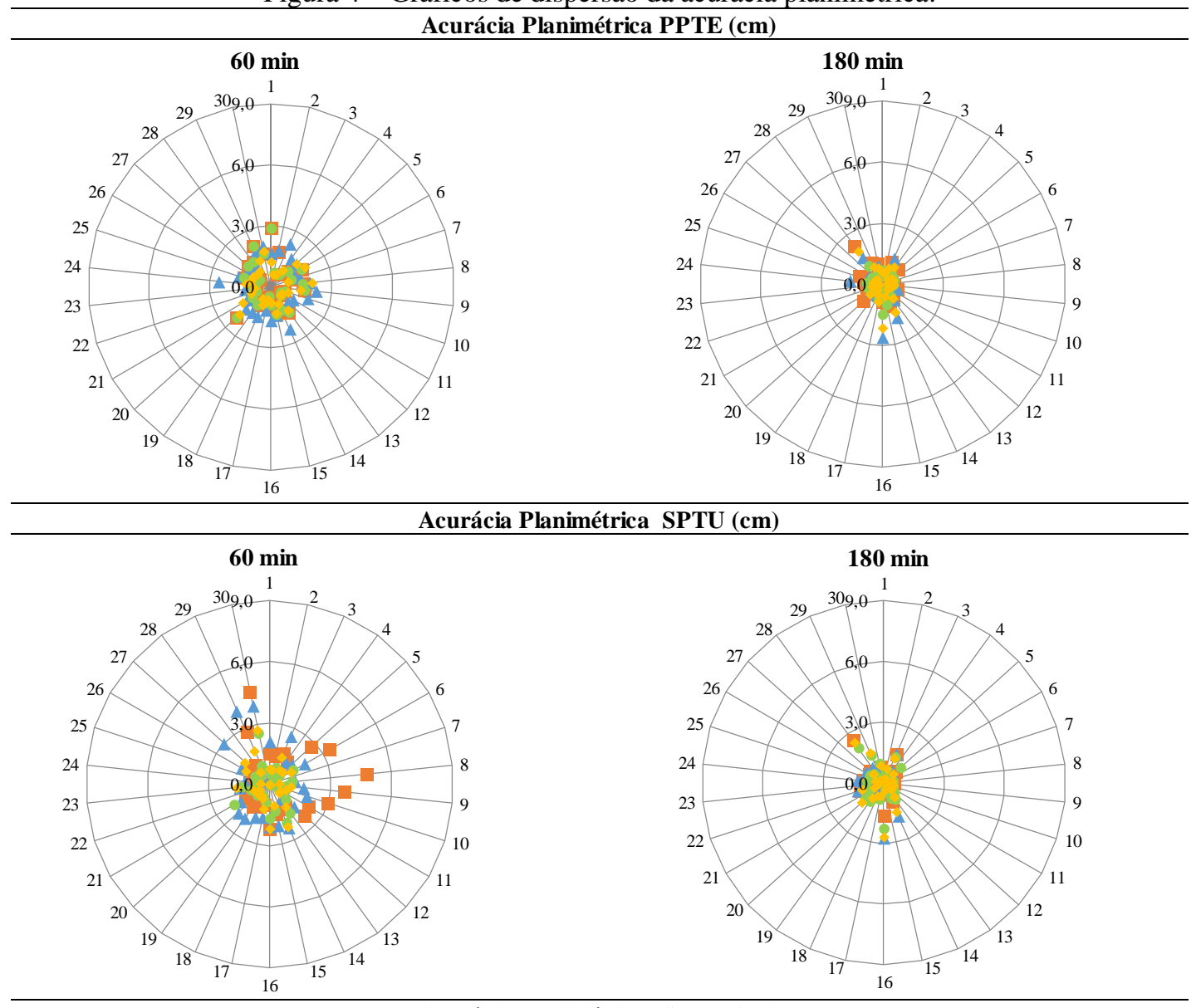

\section{Acurácia Planimétrica SPDR $(\mathbf{c m})$}
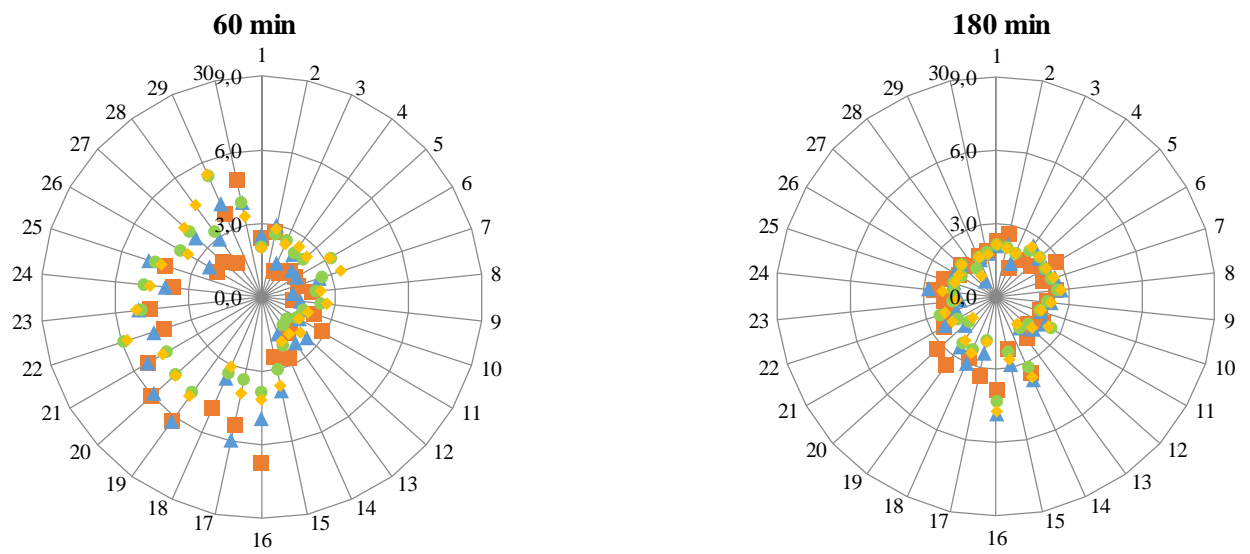

Acurácia Planimétrica MGBH $(\mathrm{cm})$
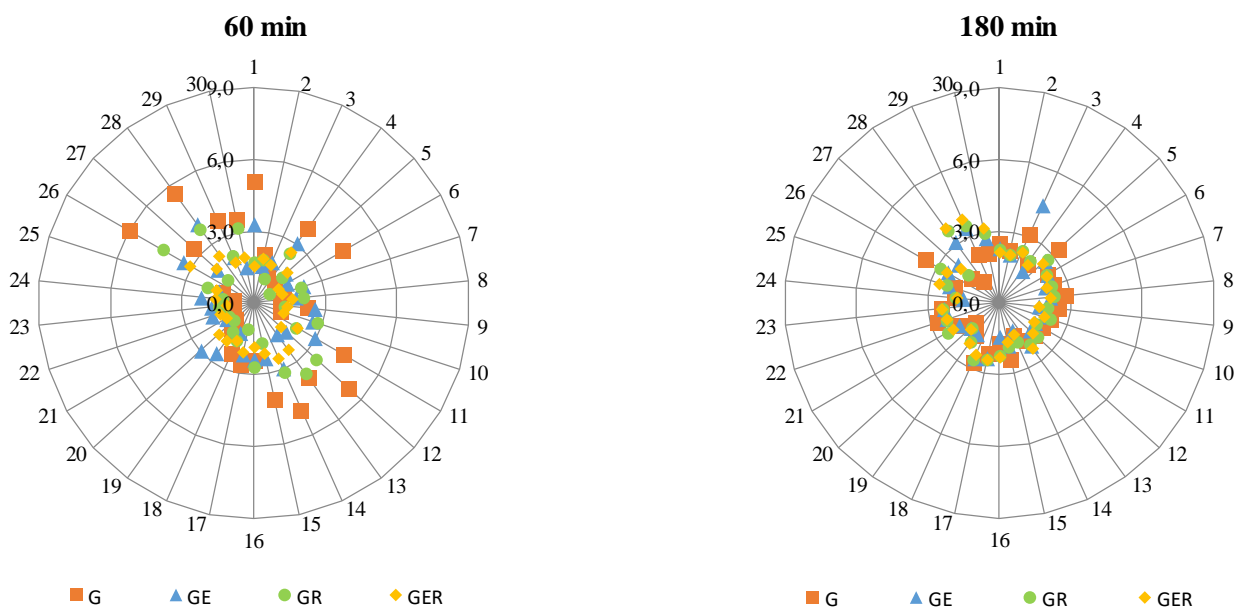

Fonte: Os autores (2020). 
A Figura 5 apresenta o histograma das ocorrências das acurácias planimétricas em classes centimétricas.

Figura 5 - Histograma de ocorrências da acurácia planimétrica em $\mathrm{cm}$.

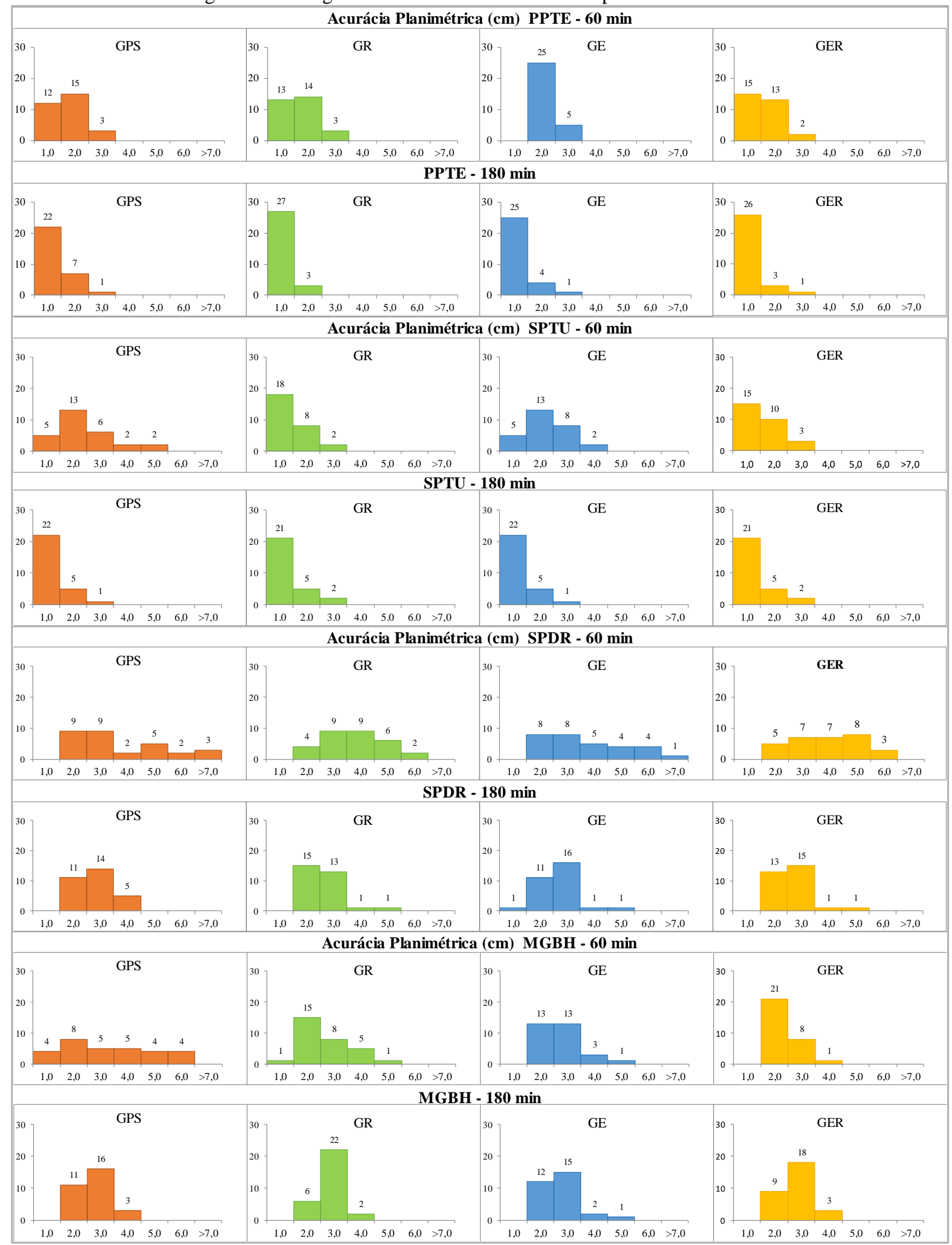

Fonte: Os autores (2020).

A Tabela 3 apresenta o Fator de Acurácia planimétricas para cada combinação de processamento e os diferentes intervalos, além dos seus respectivos totais. Destacou-se com cor verde os maiores valores e vermelho os menores valores do FA e os somatórios. 
Tabela 3 - Fator de Acurácia planimétrica.

\begin{tabular}{|c|c|c|c|c|c|}
\hline \multicolumn{6}{|c|}{ PPTE } \\
\hline Intervalo & GPS & GR & GE & GER & Total \\
\hline $60 \mathrm{~min}$ & 7,25 & 7,42 & 5,01 & 7,84 & 27,52 \\
\hline $180 \mathrm{~min}$ & 9,13 & 10,07 & 9,66 & 9,84 & 38,70 \\
\hline Total & 16,38 & 17,49 & 14,67 & 17,68 & - \\
\hline \multicolumn{6}{|c|}{ SPTU } \\
\hline Intervalo & GPS & GR & GE & GER & Total \\
\hline $60 \mathrm{~min}$ & 5,45 & 8,58 & 5,55 & 7,95 & 27,53 \\
\hline $180 \mathrm{~min}$ & 9,41 & 9,15 & 9,41 & 9,15 & 37,12 \\
\hline Total & 14,86 & 17,73 & 14,96 & 17,10 & - \\
\hline \multicolumn{6}{|c|}{ SPDR } \\
\hline Intervalo & GPS & GR & GE & GER & Total \\
\hline $60 \mathrm{~min}$ & 3,45 & 3,10 & 3,37 & 3,07 & 12,99 \\
\hline $180 \mathrm{~min}$ & 4,04 & 4,34 & 4,34 & 4,22 & 16,94 \\
\hline Total & 7,49 & 7,44 & 7,71 & 7,29 & - \\
\hline \multicolumn{6}{|c|}{ MGBH } \\
\hline Intervalo & GPS & GR & GE & GER & Total \\
\hline $60 \mathrm{~min}$ & 4,38 & 4,46 & 4,17 & 4,74 & 17,75 \\
\hline $180 \mathrm{~min}$ & 4,09 & 3,83 & 4,14 & 3,98 & 16,04 \\
\hline Total & 8,47 & 8,29 & 8,31 & 8,72 & - \\
\hline \multicolumn{6}{|c|}{ Somatório dos totais de cada combinação } \\
\hline Soma & 47,2 & 50,95 & 45,65 & 50,79 & - \\
\hline
\end{tabular}

Fonte: Os autores (2020).

Constata-se na Tabela 3 que as combinações GR e GE apresentaram três maiores valores do FA, sendo em maioria no intervalo de 180 minutos, e os menores valores foram identificados para o GPS, com três ocorrências.

Classificou-se as combinações dos sistemas baseando-se nos valores constatados no somatório dos totais de cada combinação, exposto na Tabela 3. Desta forma, pode-se verificar que a combinação que forneceu os melhores resultados é a GR, apresentando os maiores valores de FA e consequentemente as melhores acurácias. Logo em sequência, identifica-se a combinação GRE, GPS e por último o GE.

Observa-se que apesar da estação SPDR fazer uso do mesmo modelo de antena e receptor que a SPTU, foi a que obteve os menores valores de FA em toda a análise. Fato que contraria o que se espera dos resultados de duas antenas e dois receptores iguais coletando dados ao mesmo tempo.

Os resultados referentes às resultantes altimétricas das quatro estações são ilustrados através da Figura 6, 7 e da Tabela 4.

Com base nas Figuras 6 e 7, constata-se que 48,75 \% das acurácias, obtidas pela estação SPTU, são maiores ou iguais a $1,0 \mathrm{~cm}$ e apenas $24,58 \%$ para a estação PPTE. Este resultado expõe que esta estação SPTU foi responsável pelos melhores resultados em relação a todas as outras estações.

Diante dos resultados apresentados para a altimetria, verifica-se um comportamento similar a planimetria em relação ao tempo de coleta, ou seja, o aumento do intervalo de rastreio proporcionou melhores acurácias na maioria das estações. Contudo, o tempo de coleta não influenciou de forma significativa os resultados referentes a estação PPTE, principalmente, nos processamentos GPS e GER, em que ocorreu um decréscimo nos valores de FA apresentados na Tabela 4.

Tipicamente, é conhecido que antenas Choke Ring são capazes de minimizarem os efeitos de multicaminho e apresentarem maior estabilidade no centro de fase, proporcionando melhores resultados, além de serem requeridas em aplicações que exigem alta precisão (DANSKIN, BETTINGER e JORDAN, 2009). Em vista disso, esperava-se que a estação PPTE, a qual é composta por uma antena Choke Ring, apresentasse os melhores resultados em relação as demais estações. No entanto, apesar de ter apresentado as melhores acurácias na planimetria, não apresentou o mesmo comportamento para a altimetria, podendo-se constatar pelas Figuras 6 e 7 além da Tabela 4.

Nota-se pela Tabela 4 que a combinação GER apresentou cinco maiores valores do FA, nas estações PPTE, SPTU e MGBH. Já os menores valores foram identificados para a combinação GE, com quatro ocorrências nas estações PPTE e MGBH. 
Figura 6 - Gráfico de dispersão da acurácia altimétrica.

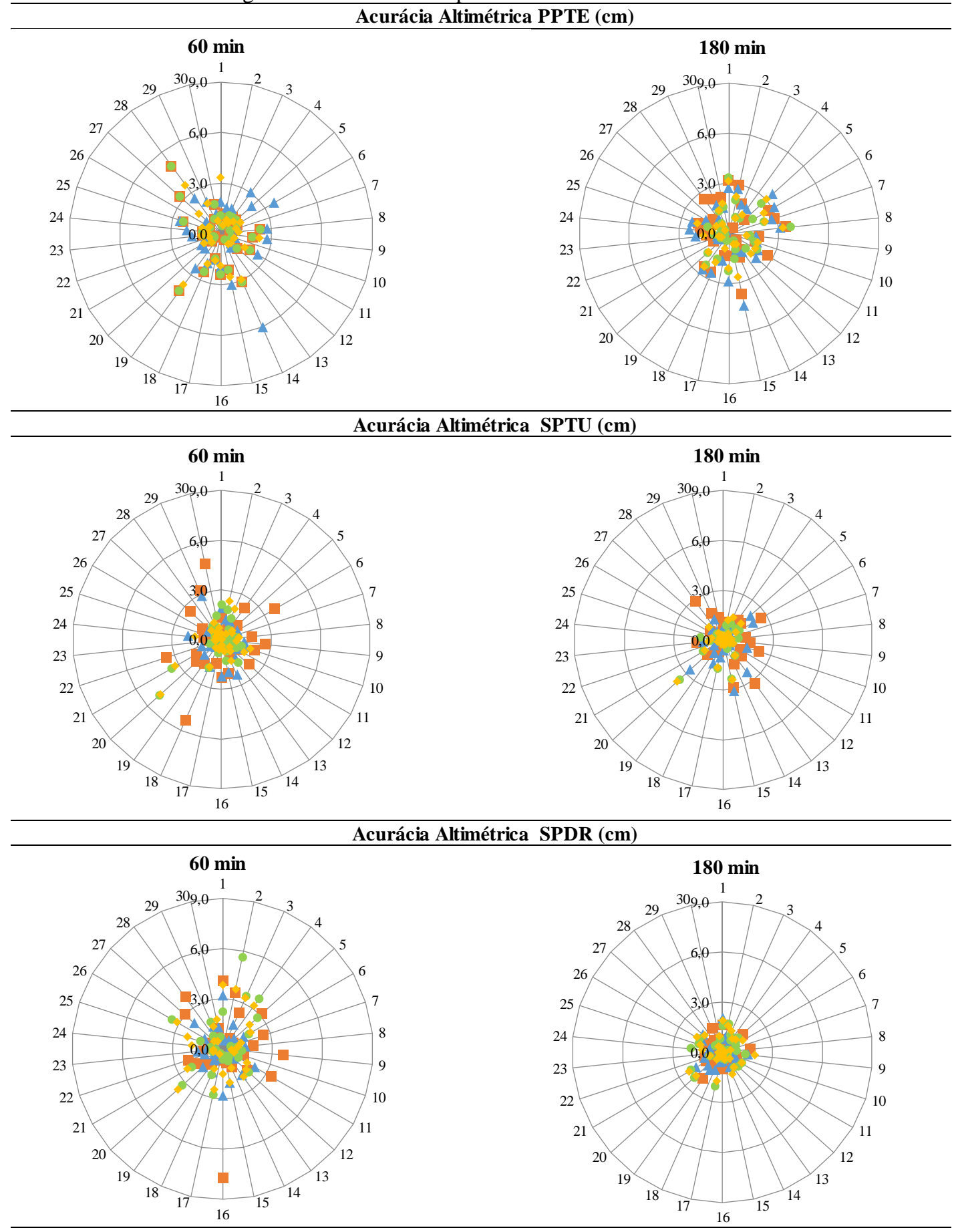

Acurácia Altimétrica MGBH $(\mathrm{cm})$
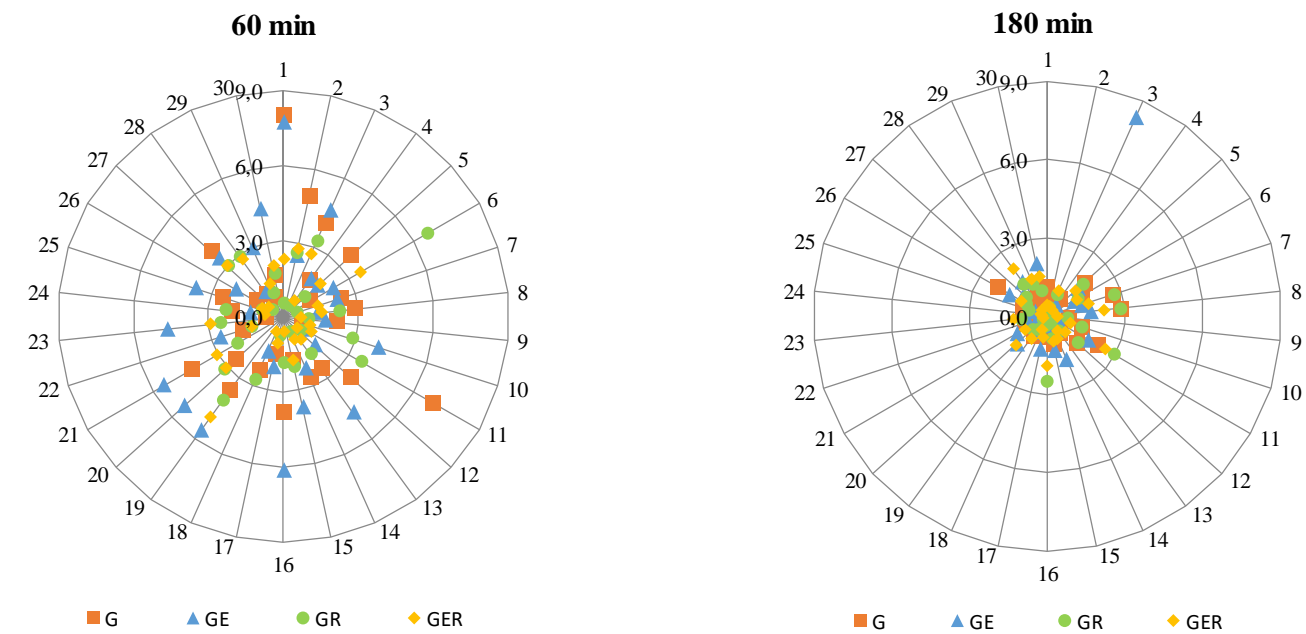

Fonte: Os autores (2020). 
A Figura 7 apresenta o histograma das ocorrências das acurácias altimétricas em classes centimétricas.

Figura 7 - Histograma de ocorrências da acurácia altimétrica.

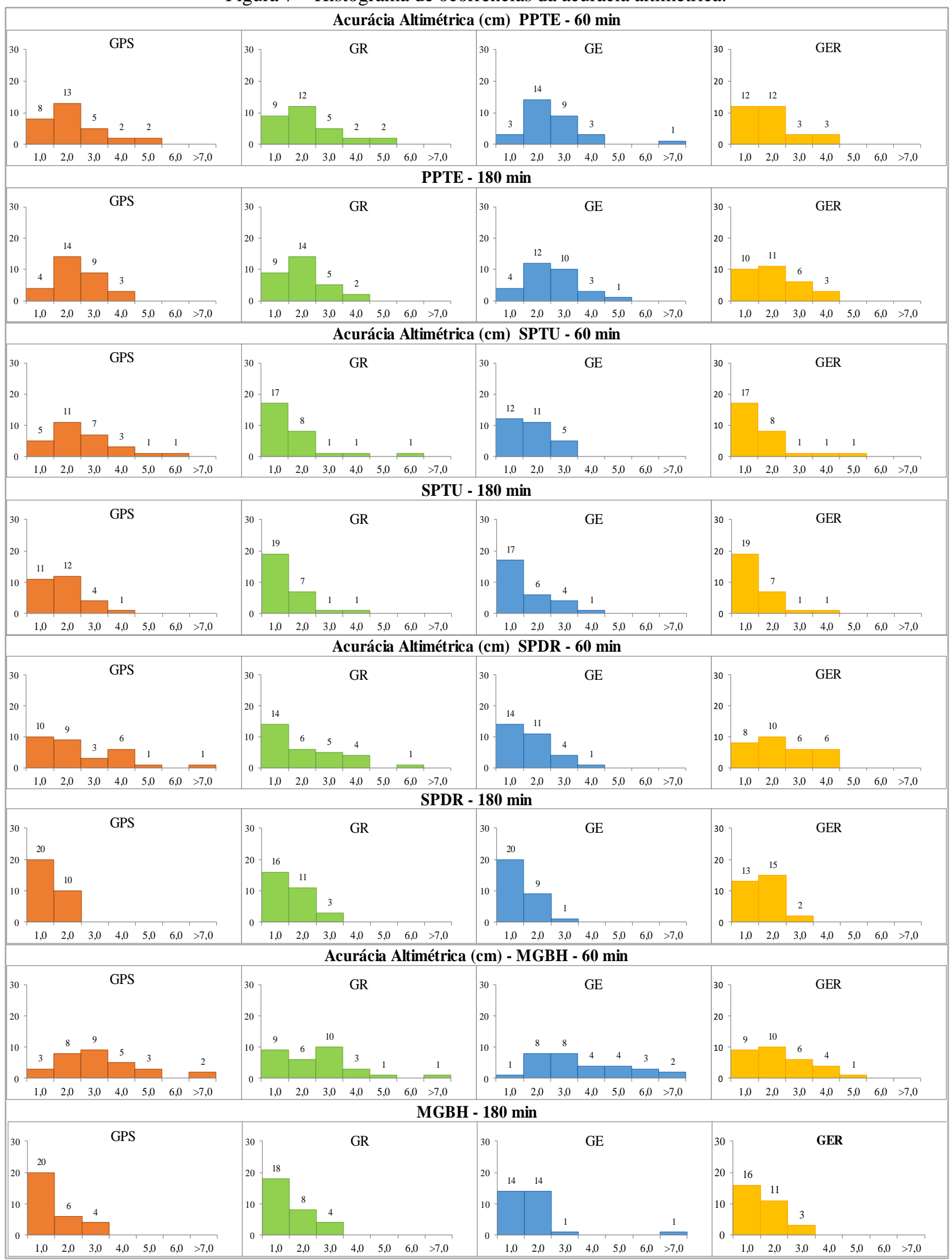

Fonte: Os autores (2020).

A Tabela 4 apresenta o Fator de Acurácia altimétrica para cada combinação de processamento e os diferentes intervalos, além dos seus respectivos somatórios. Destacou-se de verde os maiores valores e de vermelho os menores valores do FA e os somatórios. 
Tabela 4 - Fator de Acurácia altimétrica.

\begin{tabular}{|c|c|c|c|c|c|}
\hline \multicolumn{6}{|c|}{ PPTE } \\
\hline Intervalo & GPS & GR & GE & GER & Total \\
\hline $60 \mathrm{~min}$ & 6,03 & 6,21 & 4,91 & 6,98 & 24,13 \\
\hline $180 \mathrm{~min}$ & 5,21 & 6,42 & 5,05 & 6,45 & 23,14 \\
\hline Total & 11,25 & 12,63 & 9,96 & 13,43 & - \\
\hline \multicolumn{6}{|c|}{ SPTU } \\
\hline Intervalo & GPS & GR & GE & GER & Total \\
\hline $60 \mathrm{~min}$ & 5,28 & 8,24 & 7,26 & 8,25 & 29,03 \\
\hline $180 \mathrm{~min}$ & 7,04 & 8,74 & 8,17 & 8,74 & 32,70 \\
\hline Total & 12,32 & 16,98 & 15,43 & 16,99 & - \\
\hline \multicolumn{6}{|c|}{ SPDR } \\
\hline Intervalo & GPS & GR & GE & GER & Total \\
\hline $60 \mathrm{~min}$ & 6,12 & 7,01 & 7,45 & 5,83 & 26,42 \\
\hline $180 \mathrm{~min}$ & 8,84 & 7,95 & 8,78 & 7,48 & 33,05 \\
\hline Total & 14,96 & 14,96 & 16,23 & 13,31 & - \\
\hline \multicolumn{6}{|c|}{ MGBH } \\
\hline Intervalo & GPS & GR & GE & GER & Total \\
\hline $60 \mathrm{~min}$ & 4,28 & 5,81 & 3,62 & 6,08 & 19,78 \\
\hline $180 \mathrm{~min}$ & 8,60 & 8,25 & 7,58 & 7,95 & 32,38 \\
\hline Total & 11,25 & 12,63 & 9,96 & 13,43 & - \\
\hline \multicolumn{6}{|c|}{ Somatório dos totais de cada combinação } \\
\hline Soma & 49,78 & 57,20 & 51,58 & 57,16 & - \\
\hline
\end{tabular}

De forma geral, o menor valor de FA foi encontrado na estação MGBH na combinação GE (60 min 3,62) e o maior valor de FA foi na estação SPDR na combinação GPS (180 min - 8,84).

Comparando os valores encontrados no somatório dos totais de cada combinação dos sistemas utilizados, apresentados na Tabela 4, classificou-se os resultados da altimetria da mesma forma que a planimetria. Logo, pode-se constatar que os melhores resultados foram apresentados pela combinação GR, em sequência a combinação GRE, GE e por último o GPS.

\section{CONSIDERAÇÕES FINAIS E CONCLUSÕES}

Diante dos resultados obtidos neste trabalho e tomando-se como base os valores de FA, a tradicional combinação GPS/GLONASS apresentou as melhores acurácias, tanto na planimetria quanto na altimetria. Posteriormente observou-se a combinação dos sistemas GPS, GLONASS e Galileo, apresentando seu bom desempenho. Portanto, pode-se inferir que a combinação GPS/GLONASS se sobressaiu entre as demais e a combinação GPS, GLONASS e Galileo se mostrou promissora, evidenciando sua interoperabilidade no método de posicionamento PPP.

Salienta-se que a relação entre os valores das acurácias e o modelo das antenas e receptores, não apresentaram padrões conforme esperava-se. A estação PPTE, apesar de ser composta por uma antena Choke Ring, apresentou um rendimento inferior que as demais estações na análise altimétrica. Da mesma forma, as estações SPTU e SPDR apresentaram resultados não correspondentes, principalmente na planimetria.

Ao analisar o intervalo de rastreio, constata-se que o tempo de coleta influenciou nos resultados da acurácia, principalmente na altimetria. É importante frisar que o aumento do intervalo de rastreio não só melhora a acurácia, como pode minimizar algum efeito indesejável, como se observa, nas estações SPDR (planimetria) e MGBH (altimetria), em que os valores estão dispersos com 60 minutos, mas se agrupam significativamente com 180 minutos de rastreio. No entanto, em alguns casos, identificou-se situações atípicas em que a melhora nas acurácias não foi significativa e até mesmo piores, por exemplo, nas estações MGBH (planimetria) e PPTE (altimetria).

É importante destacar que a atual estrutura da constelação Galileo é limitada e encontra-se em desenvolvimento. Além disso, o processamento de observações Galileo se encontra em estágio experimental no Bernese, podendo apresentar algumas inconformidades no mesmo. Desta forma, espera-se que a partir da constelação completa do Galileo o mesmo ofereça resultados ainda mais acurados, assim como a disponibilização de softwares capazes de processá-lo individualmente e em conjunto com outros sistemas.

Em breve, mais satélites do sistema Galileo devem disponibilizar dados de navegação. Por isso, 
recomenda-se para trabalhos futuros investigar o desempenho desse sistema nos vários métodos de posicionamento, além de experimentos relacionados com a integração dos sistemas GPS, GLONASS e Galileo no posicionamento relativo.

Outra possibilidade seria analisar a qualidade do posicionamento pelo sistema Galileo em diferentes latitudes, bem como uma análise sazonal da influência da integração dos sistemas operacionais.

\section{Agradecimentos}

Esta pesquisa teve o incentivo da Universidade Federal de Viçosa através do Departamento de Engenharia Civil, curso de Pós-Graduação em Engenharia Civil que disponibilizou seus equipamentos, instalações e aplicativos computacionais, além do apoio da Coordenação de Aperfeiçoamento de Pessoal de Nível Superior - Brasil (CAPES) - Código de Financiamento 001.

\section{Contribuição dos Autores}

A metodologia foi idealizada por todos os autores, sendo o primeiro autor responsável pela investigação, validação, bem como a redação - revisão e edição (textos, revisão bibliográfica, gráficos e figuras). O segundo autor foi responsável pela análise formal e supervisão do projeto.

\section{Conflitos de Interesse}

Os autores declaram que não há conflitos de interesse.

\section{Referências}

AFIFI, A.; EL-RABBANY, A. Performance Analysis of Several GPS/Galileo Precise Point Positioning Models. Sensors. v. 15, n. 6, p. 14701-14726, 2015. DOI: 10.3390/s150614701.

ALTAMIMI, Z.; COLLILIEUX, X.; LEGRAND, J.; GARAYT, B.; BOUCHER, C. ITRF2005: A new release of the International Terrestrial Reference Frame based on time series of station positions and Earth Orientation Parameters. Journal of Geophysical Research Atmospheres. v. 112, B09401, p. 1-19. 2007. DOI: $10.1029 / 2007 J B 004949$.

ALTAMIMI, Z.; COLLILIEUX, X.; MÉTIVIER, L. Analysis and results of ITRF2008. IERS Technical Note $N^{\circ}$ 37, Frankfurt am Main: Verlag des Bundesamts für Kartographie und Geodäsie, 2012. p. 54.

ALTAMIMI, Z.; REBISCHUNG, P.; MÉTIVIER, L.; COLLILIEUX, X. ITRF2014: A new release of the International Terrestrial Reference Frame modeling nonlinear station motions. Journal of Geophysical Research: Solid Earth. v. 121, n. 8, p. 6109-6131, 2016. DOI: 10.1002/2016JB013098.

BEIDOU. BeiDou Navigation Satellite System. Systems. Disponível em: <http://en.BeiDou.gov.cn/SYSTEMS/System/>. Acesso em: set. 2018.

BRAGA, F. L. S.; DAL POZ, W. R. Mudança de referencial e atualização de coordenadas entre o IGS14 (ITRF2014) e o SIRGAS2000 (ITRF2000). In: IV SIMPÓSIO BRASILEIRO DE GEOMÁTICA (SBG2017) II JORNADAS LUSÓFONAS - CIÊNCIAS E TECNOLOGIAS DE INFORMAÇÃO GEOGRÁFICA (CTIG2017), 2017, Presidente Prudente. Anais do IV SBG 2017. p. 543-550.

BOWRING, B. R. Transformation from spatial to geographical coordinates. Survey Review, v. 23, n. 181, p. 323-327, 1976. DOI: 10.1179/sre.1976.23.181.323.

COSTA, S. M. A.; MANTOVANI, G.; SCOFANO, F.; LIMA, M. A. A.; SILVA, A. L.; MOURA JÚNIOR, N. J. Ampliação e modernização da RBMC. In: VII SIMPÓSIO BRASILEIRO DE CIÊNCIAS GEODÉSICAS E TECNOLOGIAS DA GEOINFORMAÇÃO, 2018, Recife. Anais do VII SIMGEO. p. 440-450.

DACH, R.; FRIDEZ, P. Bernese GNSS Software 5.2. Tutorial, Astronomical Institute University of Bern 
(AIUB), Bern, 2017.

DANSKIN, S.; BETTINGER, P.; JORDAN, T. Multipath Mitigation under Forest Canopies: A Choke Ring Antenna Solution. Forest Science. v. 55, n. 2, p. 109-116, 2009.

DREWES, H.; O. HEIDBACH. The 2009 Horizontal Velocity Field for South America and the Caribbean. In: KENYON S.; PACINO M.; MARTI U. (eds). Geodesy for Planet Earth. International Association of Geodesy Symposia, Springer, Berlin, v. 136, 2012. p. 657-664. DOI: 10.1007/978-3-642-20338-1_81.

EUROPEAN SPACE AGENCY (ESA). Galileo. Disponível em: <http://www.esa.int/Our_Activities/Navigation/Galileo/What_is_Galileo>. Acesso em: set. 2018.

INTERNATIONAL FEDERATION OF SURVEYORS (FIG). Technical Seminar on Reference Frame in Practice. Disponível

em: <https://www.fig.net/resources/proceedings/2016/2016_05_reference\%20frame/1_Rizos.pdf>. Acesso em: nov. 2018.

EUROPEAN GNSS SERVICE CENTRE (GSC). Constellation Information. Disponível em: <https://www.gsc-europa.eu/system-status/Constellation-Information>. Acesso em: out. 2018.

HOFMANN-WELLENHOF, B.; LICHTENEGGER, H.; COLLINS, J. GNSS - Global Navigation Satellite Systems GPS, GLONASS, Galileo, and more. New York: Springer-Verlag, 2008. DOI: 10.1007/978-3211-73017-1.

INTERNATIONAL GNSS SERVICE (IGS). [IGSMAIL-7399] Upcoming switch to IGS14/igs14.atx. Disponível em: <https://lists.igs.org/pipermail/igsmail/2016/001233.html>. Acesso em: out. 2018.

INTERNATIONAL TERRESTRIAL REFERENCE FRAME (ITRF). Transformation Parameters. Disponível em: 〈http://itrf.ensg.ign.fr/trans_para.php〉. Acesso em: jun. 2018.

JI, S.; CHEN, W.; DING, X.; CHEN, Y.; ZHAO, C.; HU, C. Potential Benefits of GPS/GLONASS/GALILEO Integration in an Urban Canyon - Hong Kong. The Journal of Navigation, v. 63, n. 04, p. 681-693. 2010. DOI: $10.1017 / \mathrm{S} 0373463310000081$.

LI, X.; ZHANG, X.; REN, X.; FRITSCHE, M.; WICKERT, J.; SCHUH, H. Precise positioning with current multi-constellation Global Navigation Satellite Systems: GPS, GLONASS, Galileo and BeiDou. Nature, Scientific Reports, v. 5, n. 8328, p. 1-14. 2015. DOI: 10.1038/srep08328.

MIKHAIL, E.; ACKERMAN, F. Observations and Least Squares. New York: IEP-A Dun-Donnelley Publisher, 1976. ISBN-10: 0819123978.

MONICO, J. F. G. Posicionamento pelo GNSS: Descrição, fundamentos e aplicações. 2.ed. São Paulo: Editora UNESP, 2008. ISBN: 9788571397880.

MONICO, J. F. M.; DAL POZ, A. P.; GALO, M.; SANTOS, M. C; OLIVEIRA, L. C. Acurácia e Precisão: Revendo os Conceitos de forma Acurada. Boletim de Ciências Geodésicas. Curitiba, v.15, n. 3, p. 469483. 2009. DOI: $10.13140 / 2.1 .1815 .8086$.

MONTENBRUCK, O.; STEIGENBERGER, P.; PRANGE, L.; DENG, Z.; ZHAO, Q.; PEROSANZ, F.; ROMERO, I.; NOLL, C.; STU“RZE, A.; WEBERI, G.; SCHMID, R.; MACLEOD, K.; SCHAERL, S. The Multi-GNSS Experiment (MGEX) of the International GNSS Service (IGS) - Achievements, prospects and challenges. Advances in Space Research. v. 59, n. 7, p. 1671-1697. 2017.

NISCHAN, T. GFZRNX - RINEX GNSS Data Conversion and Manipulation Toolbox (Version 1.05). Manual, GFZ German Research Centre for Geosciences, Alemanha, 45p. 2016.

ODIJK, D.; TEUNISSEN, P. J. G. Characterization of between-receiver GPS-Galileo inter-system biases and their effect on mixed ambiguity resolution. GPS Solutions, v. 17, n. 4, p. 521-533. 2013. DOI: 10.1007/s10291-012-0298-0.

PAN, L.; CAI, C.; SANTERRE, R.; ZHANG, X. Performance evaluation of single-frequency point positioning with GPS, GLONASS, BeiDou and Galileo. Survey Review, v. 49, p. 197-205. 2016. DOI: 10.1080/00396265.2016.1151628.

PETIT, G.; LUZUM, B. IERS Conventions (2010). IERS Technical Note $\mathrm{N}^{\circ} 36$, Frankfurt am Main: Verlag des Bundesamts für Kartographie und Geodäsie, 2010. ISBN 3-89888-989-6. 
REBISCHUNG, P.; SCHMID, R. Preparations for the IGS realization of ITRF2014. EGU General Assembly 2016, Vienna, p. 17-22. 2016.

SEEBER, G. Satellite Geodesy: foundations, methods and applications. 2 ed. New York: Walter de Gruyter, 2003. ISBN 978-3-11-020008-9.

SISTEMA DE REFERÊNCIA GEOCÊNTRICO PARA AS AMÉRICAS (SIRGAS). Definição do SIRGAS. Disponível em: <http://www.sirgas.org/pt/sirgas-definition/>. Acesso em: jun. 2018.

SETTI JÚNIOR, P. T.; ALVES, D. B. M.; GOUVEIA, T. A. F. Uso integrado dos sistemas Galileo e GPS: uma análise da acurácia no posicionamento por ponto com correções atmosféricas. Revista Brasileira de Cartografia, v. 68, n. 3, p. 595-607. Abril 2016. ISSN: 1808-0936.

SETTI JÚNIOR, P. T.; ALVES, D. B. M. Posicionamento por ponto combinado GPS e Galileo: melhorias na geometria dos satélites e na estimativa da posição. In: IV SIMPÓSIO BRASILEIRO DE GEOMÁTICA (SBG2017) II JORNADAS LUSÓFONAS - CIÊNCIAS E TECNOLOGIAS DE INFORMAÇÃO GEOGRÁFICA (CTIG2017), 2017, Presidente Prudente. Anais do IV SBG 2017. p. 558-562.

TARANTINO, E.; NOVELLI, A.; CEFALO, R.; SLUGA, T.; TOMMASI, A. Single-Frequency Kinematic Performance Comparison between Galileo, GPS, and GLONASS Satellite Positioning Systems Using an MMS-Generated Trajectory as a Reference: Preliminary Results. International Journal of GeoInformation v. 7, n. 3, p. 1-19. 2018. DOI: 10.3390/ijgi7030122.

TORGE, W. Geodesy. $3^{\mathrm{a}}$ ed. Berlin, New York: Gruyter, 2001.

UNITED NATIONS OFFICE FOR OUTER SPACE AFFAIRS (UNOOSA). Accuracy Assessment of PZ90.11 Reference Frame Based on Orbital Data Processing of GLONASS Ephemeris. Disponível em: <http://www.unoosa.org/pdf/icg/2016/icg11/wgd/13wgd.pdf>. Acesso em: dez. 2018(a).

UNITED NATIONS OFFICE FOR OUTER SPACE AFFAIRS (UNOOSA). Galileo Terrestrial Reference Frame (GTRF)-Status. Disponível em: <http://www.unoosa.org/documents/pdf/icg/2018/icg13/wgd/wgd_06.pdf >. Acesso em: dez. 2018(b).

VENTORIM, B. G; DAL POZ, W. R. Avaliação do desempenho dos sistemas GPS e GLONASS no posicionamento por ponto preciso online, combinados e individualmente. Boletim de Ciências Geodésicas, Curitiba, v. 22, n. 2, p. 264-281. Abr - jun 2016. ISSN 1982-2170.

WESTON, N. D.; SOLER, T. Rigorous Geodetic Positioning in the Americas. 8th. FIG Regional Conference 2012. Montevideo, Uruguay, p. 26-29. 2012.

\section{Biografia do autor principal}

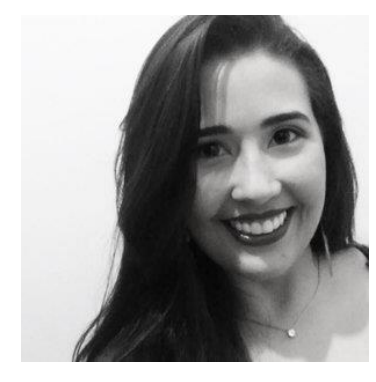

Raiane Rintielle Vaz Menezes, 23 de novembro de 1990. Possui graduação em Engenharia de Agrimensura e Cartográfica pela Universidade Federal de Viçosa (2014). Pós-Graduada em Engenharia de Segurança do Trabalho pela Universidade Cândido Mendes-UCAM (2016). Mestre em Engenharia Civil, área de Concentração em Informações Espaciais (Geodésia) e doutoranda em Engenharia Civil, área de Concentração em Informações Espaciais (Levantamentos Submarinos) pela Universidade Federal de Viçosa. Atua como Engenheira Agrimensora e Cartógrafa na área Onshore e Offshore terceirizada pela Petrobras. 